EDITORIAL AND COMMENT

\title{
Medical Scribes: Salvation for Primary Care or Workaround for Poor EMR Usability?
}

\author{
Gordon D. Schiff, MD $1,2,3$ and Laura Zucker, $M P H, M D^{4,5}$
}

'Brigham and Women's Hospital Center for Patient Safety Research and Practice, Boston, MA, USA; ${ }^{2}$ Harvard Medical School Center for Primary Care, Boston, MA, USA; ${ }^{3}$ Department of Medicine, Harvard Medical School, Boston, MA, USA; ${ }^{4}$ Family Practice Group-Sagov Center for Family Medicine, Arlington, MA, USA; ${ }^{5}$ Mount Auburn Hospital department of Internal Medicine, Cambridge, MA, USA.

J Gen Intern Med 31(9):979-81

DOI: $10.1007 / \mathrm{s} 11606-016-3788-\mathrm{x}$

() Society of General Internal Medicine 2016

I $\mathrm{n}$ this issue of the Journal, there is a small study that touches on a big set of issues. ${ }^{1}$ In fact, it offers a window into panoply of major issues that will likely decide the future of primary care. While the burden of clinical documentation is only one of a number of stresses currently facing primary care clinicians, listening to concerns in the literature and hallways, it may well be the straw that is breaking the camel's back, with reports of clinicians spending two to three extra hours each night writing notes instead of spending time with their families. ${ }^{2}$ Thus, the prospect of having a personal scribe take care of the charting and thereby allow us to spend more time better interacting with our patients as well as finish our work in a more timely way, is irresistible. As a result, medical scribes, medical scribe vendors, and practices adopting this model are rapidly proliferating, and an estimated one in five practices with an electronic medical record (EMR) currently use scribes.,

Although there is emerging data on the cost, efficiency and acceptability of medical scribes, given their growing and likely widespread future presence in our exam rooms, we know relatively little about how patients and physicians interact with and feel about scribes. Thus, the contribution of Yan et al. is a welcome glimpse into how some of the participants in the great scribe experiment feel about their work and working with each other. Given the study's small sample size, one must be cautious in generalizing to the many different settings where scribes are being used. But even among this small number of participants in this qualitative study, they found considerable variations in many of the domains studied, including workflows, interactions/relationships with patients, scribe training and duties, and impact on ability to care for patients. Overall, one gleans a mostly, but not entirely positive,

Published online July 12, 2016 picture. However, zooming the lens out can help us view some of the larger issues and implications of the use of medical scribes in primary care.

\section{SCRIBE TRAINING AND WORK ROLES}

Yan et al. highlight challenges related to staffing and training of medical scribes. Should scribes be retrofitted MAs (medical assistants) versus pre-med (or nursing/physician assistant) students eager for a chance to spend time learning medical terminology and see "real" patients? The former raises serious challenges, documented in the study, about unrealistic expectations required in potentially doing two jobs at once; the latter, while a boon for enthusiastic health care provider aspirants, means that frequent turnover, disruptions in team continuity, and investments in training fresh crops of scribes will be the norm. Finding optimal balance of work responsibilities, cost effective staffing and training will remain a challenge.

While initial fears of major detrimental impacts on exam room patient interactions and relationships appear to be allayed, there are many subtle ways the constant presence of a third person can affect medical encounters. Although all of these are not all necessarily bad (quotes we have heard at a recent workshop: "it keeps me honest, so I don't document things I didn't do"; "helps moves me along to keep up the pace of workflow"; "can look up things in the chart for me, quickly and easily" are all echoed in Yan's interviews), many issues remain unaddressed (especially when the patient is undressed!). These, including gender, language, differences among the three parties, emotionally charged moments where patients break down and/or share intimate or stressful history, and the impact on the provider-patient-scribe relationship, are deserving of further study.

\section{TAKING CLINICIANS AWAY FROM THE EMR}

Liberating the physician from the EMR during the encounter is not only good; it is also a problem. Well-designed EMRs should be serving as a rich and helpful resource for directly supporting the clinical encounter and clinician's cognitive 
work ${ }^{5}$ - reminding the clinician about the patient, past problems, history and assessments, social history and issues, as well as overdue prevention and monitoring; otherwise the clinician has to rely on memory or the scribe searching and reading this information aloud during the encounter. Doctors and patients ought to be jointly navigating through electronic information, working collaboratively and viewing the computer screen together. While this should not necessarily preclude physicians creatively re-engineering office exam room design and processes to include scribes as part of the team, having a scribe in the middle of this can at times interfere with EMR interactive work flow, real-time clinician access to helpful information, and clinician interactions with clinical decision support messages. ${ }^{3}$

\section{ENHANCING BILLING AND "UP-CODING”}

Helping to justify and capture higher reimbursement is another widely cited and documented benefit of scribes, but one that should not be accepted without more critical reflection. Here is another example of something that appears to be a plus when viewed narrowly as a way of increasing doctors' productivity and revenue, but that needs to be considered in the context of societal goals for better and more efficient primary care. To what extent do knowledgeable scribes, who become experts at meeting billing documentation requirements, serve a socially useful function vs. mainly functioning to aid individual clinicians and practices in better gaming the system? ${ }^{6}$ On the other hand, one study from a cardiology clinic did suggest that additional revenue came mainly from increased productivity and "right coding" rather than inappropriate upcoding. ${ }^{7} \mathrm{Re}-$ gardless, it is ironic that one of the consequences of EMRs, initially touted as a way to decrease medical costs, is to drive practices to bring on scribes whose documentation practices could be contributing to the overall cost of medical care.

\section{NOTE QUALITY}

In their interviews, Yan et al. found a mix of comments regarding ways scribes can enhance or detract from note quality. What is a good note? This subject is only beginning to be defined, explored and measured in the literature. With electronic notes, now that legibility is no longer an issue, most of us can subjectively invoke qualities such as completeness, accuracy (including being free from copy-paste errors), organization, succinctness, and formatting as aspects that are important to us as we read others' (and our own) notes. It is worth pondering and measuring how scribes might impact on each of these attributes. The Yan interviews suggest that real-time scribed notes can do a better job in more completely capturing information from the encounter (certainly more than a fragmentary MD note written days later). However, Yan et al. also found that scribes tended to do better at using templates and checking off boxes than they did in recording nuanced free text narratives. As a result, their ability to summarize and covey complex assessments is less predictable.

We have argued that, just as electronic medication ordering can decrease medication errors, electronic clinical documentation has great, but unrealized, potential for decreasing diagnostic errors. ${ }^{8}$ To the extent that clinical notes are relegated to mere "paperwork" to quickly document and close the encounter, rather than be conceptualized as an opportunity for clinicians to take the time to reflect on their assessment and plan, (at times better done better after the rushed pace of the clinic session is over), then something is lost when we streamline documentation via scribed notes. ${ }^{9}$ Although nothing precludes creative ways and roles scribes could play in this vision of clinical documentation, we challenge those effectively using and working with scribes to help imagine and design new workflow to enhance the quality of the note "Assessment" section. This will become increasingly important with the growth of OpenNotes (now available to nearly 8 million patients in the US) to help patients (and family caregivers) understand and reinforce clinicians' diagnostic assessments and plans based on reading notes from their own clinical encounters. ${ }^{10}$

\section{WORKAROUND RATHER THAN SOLUTION TO EMR INEFFICIENCIES}

Even though the clinician's time is extremely limited, stressed, and costly, subjecting scribes to EMR inefficiencies by delegating clinical documentation and EMR navigation to a less highly paid person does not necessarily mean we have created "efficiency." To what extent are scribes merely workarounds for suboptimal EMR design? ${ }^{3}$ In quality improvement, workarounds are understood to be ways of bypassing rather than more fundamentally addressing problems. Workarounds are often creative, innovative, and even successful, but they are suboptimal at fixing problems and can serve to mask problems, inhibit efforts to address them, or even at times introduce new problems. ${ }^{11}$ Smarter EMR interfaces and workflow designs (including smart incorporation of voice recognition) could go a long way to eliminating the reasons clinicians resort to scribes. ${ }^{12}$

In conclusion, although it appears that medical scribes are in many ways irresistible and here to stay-one of us has adopted and incorporated scribes in their practice, while the other remains more skeptical-their growth nonetheless raises broader questions about primary care work flow, staffing, clinical documentation, diagnostic assessments, provider burnout, and patient-provider- 
scribe relationships and communication. Can EMRs be transformed to better meet the needs of clinicians? Can scribes be more effectively transitioned to become more effective and skillful members of the ambulatory team? Perhaps scribes can not only help document individual encounters, but also document and provide rich insights into ways our clinical encounters, clinicians, teams, primary care systems, and electronic medical records could be improved. The comments from the 17 scribes interviewed for the Yan study represent a useful start.

Corresponding Author: Gordon D. Schiff, MD; Department of MedicineHarvard Medical School, Boston, MA, USA (e-mail: gschiff@partners.org).

\section{Compliance with Ethical Standards:}

Conflict of Interest: Dr. Schiff reports contract funding from MedAware to review data mining clinical decision support software. Dr. Zucker has no any conflicts to report.

\section{REFERENCES}

1. Yan C. Physician, scribes, and patient perspectives on clinical scribes in primary care. J Gen Intern Med. 2016. doi:10.1007/s11606-0163719-x.
2. Kuhn T, Basch P, Barr M, Yackel T. Clinical documentation in the 21st century: executive summary of a policy position paper from the American College of Physicians. Ann Intern Med. 2015;162(4):301-3.

3. Gellert GA, Ramirez R, Webster SL. The rise of the medical scribe industry: implications for the advancement of electronic health records. JAMA. 2015;313(13):1315-6.

4. Dubé $\mathbf{K}$. Re: the use of medical scribes in health care settings: a systematic review and future directions. J Am Board Fam Med. 2015;28(5):684-5.

5. Krist AH, Beasley JW, Crosson JC, et al. Electronic health record functionality needed to better support primary care. J Am Med Inform Assoc. 2014;21(5):764-71.

6. Himmelstein $\mathbf{D}$, Woolhandler $\mathbf{S}$. Quality improvement: "become good at cheating and you never need to become good at anything else. Health Aff Blog. 2015.

7. Bank AJ, Obetz C, Konrardy A, et al. Impact of scribes on patient interaction, productivity, and revenue in a cardiology clinic: a prospective study. ClinicoEcon Outcomes Res. 2013;5:399.

8. E1-Kareh R, Hasan O, Schiff GD. Use of health information technology to reduce diagnostic errors. BMJ Qual Saf. 2013. doi: 10.1136/bmjqs-2013-001884.

9. Schiff GD, Bates DW. Can electronic clinical documentation help prevent diagnostic errors? N Engl J Med. 2010;362(12):1066-9.

10. Walker J, Darer JD, Elmore JG, Delbanco T. The road toward fully transparent medical records. N Engl J Med. 2014;370(1):6-8.

11. Friedman A, Crosson JC, Howard J, et al. A typology of electronic health record workarounds in small-to-medium size primary care practices. J Am Med Inform Assoc. 2014;21(e1):e78-83.

12. Olayiwola JN, Rubin A, Slomoff T, Woldeyesus T, Willard-Grace $\mathbf{R}$. Strategies for primary care stakeholders to improve electronic health records (EHRs). J Am Board Fam Med. 2016;29(1):126-34. 